\title{
Gestão do conhecimento, internet das coisas e inovação: a relação dos temas e a intensidade de pesquisas realizadas
}

\author{
Eric de Paula Ferreira Doutorando em Sistemas de Informação e Gestão do Conhecimento. Universidade Fundação \\ Mineira de Educação e Cultura (FUMEC) - Brasil. eric.p.f@gmail.com \\ Manuel da Rocha Fiúza Branco Júnior \\ Mestrando em Sistemas de Informação e Gestão do Conhecimento. Universidade Fundação Mineira \\ de Educação e Cultura (FUMEC) - Brasil.mfiuzaj@@gmail.com \\ Paulo Isnard Doutorando em Sistemas de Informação e Gestão do Conhecimento. Universidade Fundação \\ Mineira de Educação e Cultura (FUMEC) - Brasil. ppisnard@yahoo.com.br \\ Renata de Souza França Doutoranda em Sistemas de Informação e Gestão do Conhecimento. Universidade Fundação \\ Mineira de Educação e Cultura (FUMEC) - Brasil. profrenatafranca@gmail.com \\ Armando Sérgio de Aguiar Filho Doutor em Gestão da Informação e do Conhecimento. Universidade Fundação Mineira de Educação \\ e Cultura (FUMEC) - Brasil. armando.filho@fumec.br
}

\section{RESUMO}

Os esforços de se investigar as relações entre a Gestão do Conhecimento, a Internet das Coisas e a Inovação deriva da importância que cada temática tem separadamente no cenário de constantes mudanças tecnológicas. Identificar a intensidade das possíveis relações entre estes constructos é o objetivo desta pesquisa. Essa identificação foi realizada por meio de uma análise quantitativa das publicações contidas nas bases de dados CAPES. Foi realizada também uma verificação da citação de descritores presentes nos campos título, no resumo ou nas palavras-chave dos artigos; uma análise temporal e a identificação dos principais autores e periódicos que mais tiveram publicações dos temas para alcançar um índice de impacto relevante. A escolha se deu pelos critérios de qualidade e originalidade adotados para inclusão de periódicos nessas bases. Chegou-se a um número considerável de 404.694 pesquisas científicas que apresentaram de maneira separada os temas gestão do conhecimento, internet das coisas e inovação. Porém, apenas 20 estudos os tratam de forma relacional. $O$ resultado aponta a existência de uma lacuna na integração dos temas.

Palavras-chave: Gestão do Conhecimento. Inovação. Internet das Coisas. Tecnologia da Informação.

\section{Management of knowledge, the internet of things and innovation: the relationship of the topics and the intensity of research conducted}

\begin{abstract}
The efforts to investigate the relationship between Knowledge Management, the Internet of Things and Innovation derives from the importance that each theme has separately in the current scenario of constant technological changes. To identify the intensity the possible relationships between knowledge management constructs, innovation, internet of things is the research objective. Was used a quantitative analysis of the publications in the CAPES's databases that correlate the themes. In the quantitative analysis of the works obtained in the systematic review, the analysis of the citation of descriptors present in the title fields, in the summary or in the keywords of the articles, the temporal analysis and the identification of the main authors and periodicals that had more publications of the themes to achieve the impact index of those obtained in the research. The choice was based on the criteria of quality and originality adopted to include journals in these databases A considerable number of the 404.694 scientific researches that presented separately the subjects knowledge management, internet of things and innovation. However, only 20 studies treat them relationally. The result points to the existence of a gap in the integration of themes. This result points to the existence of a gap in the integration of themes.
\end{abstract}

Keywords: Knowledge Management, Innovation, Internet of Things, Information Technology. 


\section{INTRODUÇÃO}

A proposta de uma abordagem integrativa deriva da necessidade de investigação do relacionamento entre as Temáticas de Inovação, Gestão do Conhecimento e Internet das Coisas. Nesse contexto, em que a gestão do conhecimento é vista como o objeto de ligação entre essas temáticas, torna-se fundamental definir o conhecimento para entender qual é a dinâmica dessa relação.

No recorte proposto por esse artigo, o conhecimento é a informação presente na mente e que pode ser gerenciado em favor da organização, mesmo sabendo que a informação individualizada pode ou não ser útil. Segundo Davenport e Prusak (1998) da gestão do conhecimento resultam diferentes processos de captura e compartilhamento de conhecimento. Processos esses, que podem conectar variadas temáticas, dentre elas a Inovação e Internet das Coisas.

A Inovação é a principal ferramenta utilizada para alavancar o crescimento das organizações, aumentando sua produtividade e competitividade. Porém, inserir a empresa dentro de uma cultura inovadora não é algo tão fácil como pode parecer (FREEMAN; SOLET, 1982; BAREGHEH; ROWLEY; SAMBROOK, 2009). A medida que as organizações aprofundam suas raízes na economia do conhecimento, novos mercados são criados, sobretudo em produtos e serviços intensivos em TI, pois, são capazes de oferecer suporte a várias atividades de inovação, especialmente aquelas que se baseiam em informação e conhecimento (TIGRE, 2006; PINHEIRO, 2011).

Nesse contexto, a aquisição de dados é um fator crucial, pois, sistemas de coleta de dados baseados em atividade humana já não conseguem atender às necessidades organizacionais. A computação em nuvem (Cloud Computing), vem prover a infraestrutura virtual que integrará dispositivos de monitoramento e armazenagem, ferramentas analíticas, plataformas de visualização e entrega aos usuários. O conceito de Internet das coisas precisa de desenvolvimento, conectando cada objeto do dia a dia e incorporando inteligência ao ambiente (GUBBI et al., 2013). Esse conceito afeta produtos e serviços que deverão se inovar para incorporá-los.

O estudo busca responder quais as possíveis relações entre os constructos Gestão do Conhecimento, Inovação e Internet das Coisas. Essa identificação será realizada por meio de uma análise quantitativa das publicações, bem como a ocorrência de palavras, periódicos e áreas de pesquisa que mais relacionam as temáticas. O trabalho seguirá a seguinte estrutura: (i) (ii) (iii) revisão teórica contemplando conceitos de Gestão do Conhecimento, Internet das Coisas e Inovação; (iv) metodologia da pesquisa; (v) apresentação dos resultados da revisão sistemática; (vi) resultado da análise temporal e (vii) considerações finais.

\subsection{Objetivo geral}

O objetivo da pesquisa é identificar a intensidade das possíveis relações entre os constructos Gestão do Conhecimento, Inovação e Internet das Coisas.

\subsection{Objetivos específicos}

Para alcançar o objetivo geral proposto, tem-se como objetivos específicos:

a) Identificar a intensidade de pesquisas dos constructos isoladamente: Gestão do Conhecimento, Internet das Coisas e Inovação.

b) Identificar a intensidade de pesquisa por pares: Gestão do Conhecimento $x$ Internet das Coisas; Gestão do Conhecimento x Inovação; Internet das Coisas x Inovação.

c) Identificar fatores caracterizadores das pesquisas que relacionem de maneira conjunta Gestão do Conhecimento, Internet das Coisas e Inovação. 


\section{GESTÃO DO CONHECIMENTO}

As relações entre dados, informação e conhecimento são intrínsecas. Embora sejam conceitos complementares, os níveis de importância estão em patamares distintos no ambiente organizacional. Segundo Davenport e Prusak (1998) identificar as diferenças entre os três conceitos é de grande importância para as organizações, pois, os processos decisórios dependem da aplicação correta e bem definida dos mesmos.

Paiva (2012) assume que a gestão do conhecimento não terá sentido se não houver a diferenciação entre conhecimento, dados e informações. $O$ autor explica que dado é a representação de um fato de forma formalizada, capaz de ser comunicado por algum processo. Já para Davenport e Prusak (1998), dado pode ser definido como a coleção de fatos que são específicos de alguns eventos. $O$ dado não tem propósito quando está separado do contexto.

A informação pode ser definida como significado que um humano atribui aos dados por meio das convenções conhecidas usadas em sua representação. A combinação de dados fornece informações contextualizadas, ou seja, dados podem ser convertidos em informação quando os mesmo são utilizados para um propósito específico (DAVENPORT; PRUSAK, 1998). Algo que parece indiscutível é que as organizações vêm apoiando cada vez mais o uso desses recursos para possibilitar maior facilidade no trabalho.

É importante notar que a conversão de dados para informação só tem o valor reconhecido em virtude do atual nível de difusão da tecnologia. Além disso, deve-se considerar que o uso comercial da web tornou possível a oferta de determinados serviços (TICs), que seria o primeiro passo da globalização, tida como um dos maiores avanços tecnológicos descritos por Toffler (1980) na Terceira Onda.

O conhecimento é uma informação personalizada possuída na mente dos indivíduos, que é relacionado à cognição e julgamentos. Na gestão do conhecimento, além dos autores seminais (NONAKA TAKEUCHI, 1997; DAVENPORT; PRUSAK, 1998; CHOO, 2003), identifica-se um número de perspectivas evolutivas de conhecimento e implicações para seu papel e sua gestão, dentre as mais significativas pode-se citar: permitir aos usuários acessar informações de uma forma inteligível (PAIVA, 2012).

A relação entre informação e conhecimento deve ser tratada como um fenômeno complexo e, no caso deste trabalho, a proposta é a contextualização da gestão do conhecimento em uma abordagem gerencial que pode ser analisada sob duas perspectivas: uma científica e outra social.

$\mathrm{Na}$ perspectiva científica o foco direciona-se especialmente na tecnologia, na informação e no conhecimento, enquanto o conhecimento é visto como um campo ou disciplina separada e a gestão do conhecimento como uma dimensão adicional. Já na perspectiva social o conhecimento é entendido como um processo, imerso também em processos informais (NONAKA; TAKEUCHI, 1997). A compreensão subjetiva trata o conhecimento como dependente das pessoas, por meio de suas experiências.

Neste ponto de vista, o conhecimento é um processo eficaz, que possibilita a transformação de um aprendizado não reflexivo em uma prática habitual, explicitando as regras e condutas que norteiam as atividades das rotinas e facilitando o surgimento de novos conhecimentos (TSOUKAS; VLADIMIROU, 2001).

\section{INTERNET DAS COISAS}

O termo Internet das Coisas (Internet of Things - IOT) foi utilizado pela primeira vez em 1999 por Kevin Ashton, justamente conectando a nova ideia à época do uso de radio frequency identification system (RFID) na cadeia de suprimento da P\&G com a Internet. RFID é uma tecnologia que usa comunicações de rádio sem fio para identificação exclusiva de pessoas ou ativos e permite rastrear itens por meio de ondas de rádio frequência e microchip (ASHTON, 2009).

A falta de especialistas em tecnologia, a integração com sistemas ou processos atuais e a dificuldade de integração com os sistemas de automação existentes são barreiras aos sistemas RFID. Além disso, a restrição de custos se torna uma razão básica por trás da não implementação dessa tecnologia em larga escala. $\mathrm{A}$ Internet das Coisas pode ser considerada como a rede mundial de objetos interligados de endereço exclusivo, com base em protocolos de comunicação padrão (SINGER, 2012). 
O conceito de "Coisa" tem evoluído junto à tecnologia, mas, o principal objetivo é obter informações úteis para sistemas computadorizados sem a intervenção humana. Para isso, é necessária uma evolução na rede de objetos interconectados que colheriam informações do ambiente (sensoriamento) e interagiriam com o mundo físico (atuação, comando e controle) (SINGER, 2012; ASHTON, 2009).

Também são necessários novos padrões para a Internet que permitam serviços de transferência, análise e aplicação da informação além da comunicação.

Gubbi et al. (2013) apresentam uma classificação das "coisas" em quatro áreas de aplicação e seus usuários finais: i) Casa, composto pela relação de saúde, entretenimento e eletrodomésticos. ii) Transporte: representado pela logística, tráfego, estacionamento, serviços de emergência e estradas; iii) Comunidade: composta por fábricas, varejo, meio ambiente, inteligência de negócios, vigilância e mediação inteligente; e, iv) Nacional: sendo os usuários serviços públicos, infraestrutura, smart grid, monitoramento remoto e defesa.

Percebe-se então, que o desenvolvimento da internet das coisas é crescente e promissor e pode ser vista como uma inovação tecnológica. Em 2013, 9 bilhões de dispositivos estavam conectados à Internet e as projeções indicam que serão 24 bilhões em 2020. Esses dispositivos significam US\$1,3 trilhões em receitas para operadoras de redes móveis atendendo a setores como saúde, setor automotivo, serviços públicos e os consumidores em geral (FERRAZ, 2016).

\section{INOVAÇÃO}

A inovação é destacada na literatura como diferencial competitivo para as empresas, capaz de proporcionar um caminho para o crescimento e liderança de um produto ou serviço (MUYLDER et al., 2015). Segundo Terra (2012) definir o seu posicionamento para inovar é o primeiro passo, pois, sem esse alinhamento uma parte crucial dos esforços de inovação na empresa pode ser desperdiçada, podendo essa não atingir os objetivos esperados.

Diferentes são as definições sobre inovação e quais estratégias seguir para potencializar a geração da mesma. Schumpeter (1981) propõe que a inovação é a capacidade da empresa de superar a concorrência perfeita, estabelecendo, ao criar um novo mercado para os seus produtos. Tidd, Bessant e Pavitt (2008), seguindo a visão mais economicista de Shumpeter (1981), defendem que a inovação é algo novo que agregue valor social ou riqueza, ao desenvolvimento de novos valores e que mantêm ou aumentam a posição competitiva de uma empresa podendo ser classificados em quatro grupos: de produto, de processo, de posição ou de paradigma.

Nord e Tucker (1987) trazem uma abordagem mais tecnológica para a definição da inovação. Segundo os autores inovação é um produto relacionado a novas tecnologias. Em complemento e com a mesma visão, para Baglieri (2003) é a introdução de novas tecnologias em um produto ou no processo de fabricação a fim de aprimorar seu desempenho e usabilidade ou minimizar seu custo. Essa abordagem é interessante porque, segundo Lopes e Barbosa (2008) muitas pesquisas sobre o tema inovação são relacionados à tecnologia.

Contudo, pode-se considerar a inovação como um processo que se inicia com uma nova ideia, passa pela seleção de soluções aplicadas e em seguida pelo desenvolvimento da solução que visa transformá-la em novo produto, processo ou serviços (SOUZA et al., 2011) e iniciativas relevantes sobre inovação no setor de serviços surgiram.

No Brasil, essas iniciativas foram desenvolvidas na segunda metade da década de 2000, encetadas por duas instituições produtoras de estatísticas econômicas, o IBGE (Instituto Brasileiro de Geografia e Estatística) com a Pintec (Pesquisa de Inovação) e o IPEA (Instituto de Pesquisa Econômica Aplicada) com o estudo "Estrutura e dinâmica de serviços no Brasil" (BERNARDES; ANDREASSI, 2007).

Ao tempo que as organizações aprofundam suas raízes na economia do conhecimento, novos nichos de mercados são criados, especialmente em produtos e serviços intensivos em Tecnologias da Informação e Comunicação (TIGRE, 2006) e segundo Muylder et al. (2015), junto com a capacidade de gerir a inovação, surge também a necessidade de mensurá-la, pois, qualquer esforço deve ser medido a fim de verificar o quanto está sendo válido. 
Para Tidd, Bessant e Pavitt (2008) gerir a inovação compreende a capacidade de transformar incertezas em conhecimento, mas isso só é possível mobilizando recursos no sentido de reduzir essas incertezas. Desta forma, pode-se dizer que a gestão da inovação consiste em aprender a encontrar a solução mais apropriada para o problema de gerenciar o processo inovação de maneira eficiente, fazendo-o pelos meios indicados, levando em conta as circunstâncias em que as empresas se encontram.

\section{METODOLOGIA}

A metodologia de pesquisa indica o que foi pesquisado e as etapas de condução do estudo, desde a sua concepção até as conclusões. A escolha da metodologia correta é um fator crítico para o sucesso de qualquer pesquisa. Para o presente estudo foi desenvolvida uma revisão sistemática, de natureza aplicada e com objetivos de caráter exploratório.

A pesquisa caracteriza-se como descritiva de abordagem quantitativa. As análises quantitativas são mais adequadas para apurar e realizar uma classificação, pois, utiliza diferentes técnicas estatísticas para quantificar dados. Realiza-se para compreender e enfatizar o raciocínio lógico e todas as informações que se possam mensurar sobre as experiências humanas (YAMAKAWA et al., 2014).

O levantamento iniciou pela busca em bases de dados científicas dos termos chaves: Gestão do Conhecimento, Tecnologia da Informação, Internet das Coisas e Inovação. Foi realizada também uma análise temporal e de descritores para identificar a intensidade de ocorrência de palavras, principais autores, periódicos e áreas de concentração que relacionem o tema.

\subsection{Revisão sistemática da literatura}

Segundo Perissé, Gomes e Nogueira (2001) a revisão sistemática da literatura é a aplicação de estratégias cientificas que viabiliza a seleção de estudos relevantes sobre um determinado tópico. O processo de revisão sistemática da literatura se diferencia dos demais métodos pela sua rigorosidade, relacionando as evidências de vários artigos científicos e com base em sua síntese busca deixar evidente possíveis vieses que o pesquisador possa ter na análise sobre um tema especifico (GALVÃO; SAWADA; TREVISAN, 2004).

A revisão sistemática da literatura pretende responder a uma pergunta claramente formulada utilizando métodos sistemáticos e explícitos para identificar, selecionar e avaliar criticamente pesquisas relevantes, e coletar e analisar dados de estudos pertencentes à revisão (CASTRO et al., 2002).

Diante da diversidade de conceitos que envolvem a Gestão do Conhecimento, a Internet das Coisas e a Inovação, o presente estudo buscou estabelecer o vínculo entre os três constructos e apresentar uma visão integrativa das publicações que abordam os temas de maneira conjunta.

Primeiro foram buscados os textos na base CAPES que apresentassem nos campos de pesquisa título, palavras-chave e resumo, os temas de maneira isolada: Gestão do Conhecimento, Internet das Coisas ou Inovação. Posteriormente, relacionou-se os temas em pares: Gestão do Conhecimento e Internet das Coisas; Gestão do Conhecimento e Inovação; Internet das Coisas e Inovação. Por fim, buscou-se a relação dos temas Gestão do Conhecimento, Internet das Coisas e Inovação.

As bases de dados CAPES, foram criadas com o propósito de compilar, em um domínio único, publicações cientificas a fim de facilitar os esforços de pesquisa dos pesquisadores brasileiros. As publicações disponíveis nas bases de dados CAPES são respaldadas por qualidade e originalidade, devido aos critérios adotados para inclusão de periódicos.

Ao selecionar as bases mais relevantes, foi considerado que cada base de dados tem sua peculiaridade. Assim, foram definidas prioritariamente a área de Ciências Sociais Aplicadas e Multidisciplinar, pois, ambas abrangem publicações nas áreas de direito, administração, economia, arquitetura, planejamento urbano, demografia, ciência da informação, museologia, comunicação e turismo. 


\section{RESULTADOS DA REVISÃO SISTEMÁTICA DE LITERAURA}

Nessa sessão são apresentados os resultados das análises frente as temáticas da pesquisa. Foram avaliados os termos na língua inglesa e portuguesa, da seguinte forma: análise individual dos termos, análise por pares e análise das três temáticas envolvidas no estudo.

\subsection{Análise individual das temáticas: gestão do conhecimento, internet das coisas e inovação}

Por meio da busca dos constructos de maneira isolada, percebeu-se que há uma vasta literatura que aborda os temas separadamente, conforme visto nos quadros 1 , 2 e 3 . Na pesquisa realizada no dia 26 de setembro de 2017, por meio das chaves de busca, encontrou-se 404.694 documentos contendo um dos termos gestão do conhecimento, internet das coisas ou inovação.

Quadro 1- Pesquisa utilizando o termo Gestão do Conhecimento

\begin{tabular}{|l|l|}
\hline Realizada em: & 26/09/2017 \\
\hline Termos de pesquisa: & "Gestão do conhecimento" OR "Knowledge management" \\
\hline Chave de busca: & $\begin{array}{l}\text { TITLE-ABS-KEY ("Gestão do conhecimento" OR "Knowledge management") } \\
\text { AND (LIMIT-TO (DOCTYPE,"ar")) }\end{array}$ \\
\hline Período: & Sem restrições \\
\hline Campos de Pesquisa: & Título, palavras-chave e resumo \\
\hline Tipo de Publicação: & Só artigos com textos completos \\
\hline Nível da Publicação: & Sem restrições \\
\hline Periódicos: & Bases CAPES \\
\hline Idiomas: & Sem restrições \\
\hline Retornos: & 60.917 documentos \\
\hline
\end{tabular}

Fonte: dados da pesquisa (2017)

Quadro 2- Pesquisa utilizando o termo Internet das Coisas

\begin{tabular}{|l|l|}
\hline Realizada em: & 26/09/2017 \\
\hline Termos de pesquisa: & "Internet das coisas" OR" Internet of things" \\
\hline Chave de busca: & $\begin{array}{l}\text { TITLE-ABS-KEY ("Internet das coisas" OR "Internet of things") AND (LIMIT-TO } \\
\text { (DOCTYPE."ar")) }\end{array}$ \\
\hline Período: & Sem restrições \\
\hline Campos de Pesquisa: & Título, palavras-chave e resumo \\
\hline Tipo de Publicação: & Só artigos com textos completos \\
\hline Nível da Publicação: & Sem restrições \\
\hline Periódicos: & Bases CAPES \\
\hline Idiomas: & Sem restrições \\
\hline Retornos: & 24.667 documentos \\
\hline
\end{tabular}

Fonte: dados da pesquisa (2017)

Quadro 3- Pesquisa utilizando o termo Inovação

\begin{tabular}{|l|l|}
\hline Realizada em: & 26/09/2017 \\
\hline Termos de pesquisa: & "Inovação" OR" Innovation" \\
\hline Chave de busca: & TITLE-ABS-KEY ("Inovação" OR "Innovation") AND (LIMIT-TO (DOCTYPE,"ar")) \\
\hline Período: & Sem restrições \\
\hline Campos de Pesquisa: & Título, palavras-chave e resumo \\
\hline Tipo de Publicação: & Só artigos com textos completos \\
\hline
\end{tabular}




\begin{tabular}{|l|l|}
\hline Nível da Publicação: & Sem restrições \\
\hline Periódicos: & Bases CAPES \\
\hline Idiomas: & Sem restrições \\
\hline Retornos: & 319.110 documentos \\
\hline
\end{tabular}

Fonte: dados da pesquisa (2017)

Nota-se que estudos sobre internet das coisas são os mais encontrados na base, seguido de gestão do conhecimento e inovação, demonstrando a importância do desenvolvimento tecnológico para as organizações e a sociedade.

\subsection{Análise relacional por pares das temáticas: gestão do conhecimento, internet das coisas e inovação}

Ao relacionar os termos Gestão do Conhecimento, Internet das Coisas e Inovação por pares, percebeuse uma redução significativa no número de pesquisas retornadas. A relação dos temas gestão do conhecimento e internet das coisas, retornou 152 estudos, conforme o Quadro 4.

Quadro 4 - Pesquisa Gestão do Conhecimento x Internet das Coisas

\begin{tabular}{|l|l|}
\hline Realizada em: & $26 / 09 / 2017$ \\
\hline Termos de pesquisa: & $\begin{array}{l}\text { ("Knowledge management" OR "Gestão do conhecimento") AND ("Internet } \\
\text { das coisas" OR "Internet of things") }\end{array}$ \\
\hline Chave de busca: & TITLE-ABS-KEY (("Knowledge management" OR "Gestão do conhecimento") \\
& $\begin{array}{l}\text { AND ("Internet das coisas" OR "Internet of things")) AND (LIMIT-TO } \\
\text { (DOCTYPE,"ar")): }\end{array}$ \\
\hline Período: & Sem restrições \\
\hline Campos de Pesquisa: & Título, palavras-chave e resumo \\
\hline Tipo de Publicação: & Só artigos com textos completos \\
\hline Nível da Publicação: & Sem restrições \\
\hline Periódicos: & Bases CAPES \\
\hline Idiomas: & Sem restrições \\
\hline Retornos: & 152 documentos \\
\hline
\end{tabular}

Fonte: dados da pesquisa (2017)

Na relação de gestão do conhecimento e inovação, obteve-se 120 pesquisas como retorno, conforme o Quadro 5.

Quadro 5- Pesquisa Gestão do Conhecimento x Inovação

\begin{tabular}{|l|l|}
\hline Realizada em: & $26 / 09 / 2017$ \\
\hline Termos de pesquisa: & $\begin{array}{l}\text { ("Knowledge management" OR "Gestão do conhecimento") AND ("Inovação" } \\
\text { OR "Innovation") }\end{array}$ \\
\hline Chave de busca: & $\begin{array}{l}\text { TITLE-ABS-KEY (("Knowledge management" OR "Gestão do conhecimento") } \\
\text { AND ("Inovação" OR "Innovation")) AND (LIMIT-TO (DOCTYPE,"ar")) }\end{array}$ \\
\hline Período: & Sem restrições \\
\hline Campos de Pesquisa: & Título, palavras-chave e resumo \\
\hline Tipo de Publicação: & Só artigos com textos completos \\
\hline Nível da Publicação: & Sem restrições \\
\hline Periódicos: & Bases CAPES \\
\hline Idiomas: & Sem restrições \\
\hline Retornos: & 120 documentos \\
\hline
\end{tabular}

Fonte: dados da pesquisa (2017) 
Ao pesquisar os estudos que relacionam inovação e internet das coisas, foram retornados 776 estudos, conforme o Quadro 6.

Quadro 6- Pesquisa Inovação x Internet das Coisas

\begin{tabular}{|l|l|}
\hline Realizada em: & $26 / 09 / 2017$ \\
\hline Termos de pesquisa: & $\begin{array}{l}\text { ("Inovação" OR "Innovation") AND ("Internet das coisas" OR " Internet of } \\
\text { things") }\end{array}$ \\
\hline Chave de busca: & $\begin{array}{l}\text { TITLE-ABS-KEY (("Inovação" OR "Innovation") AND ( "Internet das coisas" OR } \\
\text { "Internet of things" )) AND (LIMIT-TO (DOCTYPE,"ar")) }\end{array}$ \\
\hline Período: & Sem restrições \\
\hline Campos de Pesquisa: & Título, palavras-chave e resumo \\
\hline Tipo de Publicação: & Só artigos com textos completos \\
\hline Nível da Publicação: & Sem restrições \\
\hline Periódicos: & Bases CAPES \\
\hline Idiomas: & Sem restrições \\
\hline Retornos: & 776 documentos \\
\hline
\end{tabular}

\subsection{Análise relacional das temáticas: gestão do conhecimento, internet das coisas e inovação}

Para atender ao objetivo desse estudo de verificar os estudos que relacionam Gestão do Conhecimento, Internet das Coisas simultaneamente, foi realizada uma busca integrada com as três temáticas, conforme o Quadro 7.

Quadro 7- Pesquisa Gestão do Conhecimento x Internet das Coisas x Inovação

\begin{tabular}{|l|l|}
\hline Realizada em: & $26 / 09 / 2017$ \\
\hline Termos de pesquisa: & ("Gestão do conhecimento" OR "Knowledge management") \\
& $\begin{array}{l}\text { AND ("Inovação" OR "Innovation") AND ("Internet das coisas" OR "Internet of } \\
\text { things") }\end{array}$ \\
\hline Chave de busca: & $\begin{array}{l}\text { TITLE-ABS-KEY (("Gestão do conhecimento" OR "Knowledge management") } \\
\text { AND ("Inovação" OR "Innovation") AND ("Internet das coisas" OR" Internet of } \\
\text { things")) AND (LIMIT-TO (DOCTYPE,"ar")) }\end{array}$ \\
\hline Período: & Sem restrições \\
\hline Campos de Pesquisa: & Título, palavras-chave e resumo \\
\hline Tipo de Publicação: & Só artigos com textos completos \\
\hline Nível da Publicação: & Sem restrições \\
\hline Periódicos: & Bases CAPES \\
\hline Idiomas: & Sem restrições \\
\hline Retornos: & 20 documentos \\
\hline
\end{tabular}

Fonte: dados da pesquisa (2017)

Percebe-se que as pesquisas que relacionam as temáticas de gestão do conhecimento, internet das coisas e inovação ainda são incipientes. Retornaram apenas 20 pesquisas que relacionam as três temáticas e apresentam de maneira simultânea essas abordagens. 


\section{DESCRIÇÃO DOS RESULTADOS POR ANÁLISE TEMPORAL E AS PRINCIPAIS CARACTERÍSTICAS DAS PUBLICAÇÕES}

Após a análise de todos os cenários fica evidente a existência de uma lacuna na integração dos temas Gestão do Conhecimento, Internet das coisas e Inovação. Na pesquisa, buscou-se afunilar e prosseguir a análise dos constructos mais relevantes, levando em consideração a premissa da gestão do conhecimento como papel integrador dos temas, pois, segundo Perrotti e Vasconcellos (2005), a geração do conhecimento é encontrada em organizações que possuem estruturas mais flexíveis, temporárias e autônomas e que facilitem a integração interfuncional e interdisciplinar.

A pesquisa se propôs a fazer a análise quantitativa dos trabalhos obtidos na revisão sistemática começando pela análise da citação de descritores presentes nos campos título, no resumo ou nas palavraschave dos artigos, objetivando a identificação da maior densidade de palavras-chave. Logo após, foi realizada uma análise temporal e, posterirormente, a identificação dos principais autores e dos periódicos que mais tiveram publicações dos temas para alcançar o índice de impactos obtidos na pesquisa.

\subsection{Contagem de palavras e densidade de termos}

Segundo Guedes e Borschiever (2005) por meio da lei de Zipf consegue-se fazer a constatação da frequência das palavras dentro de um texto. A lei estabelece que as palavras mais citadas tendem a possuir maior relação com o assunto do documento. Ao realizar a contagem das palavras nos 20 textos selecionados, é encontrado o resultado apresentado na Figura 1.

Figura 1 - Contagem de palavras dos textos selecionados

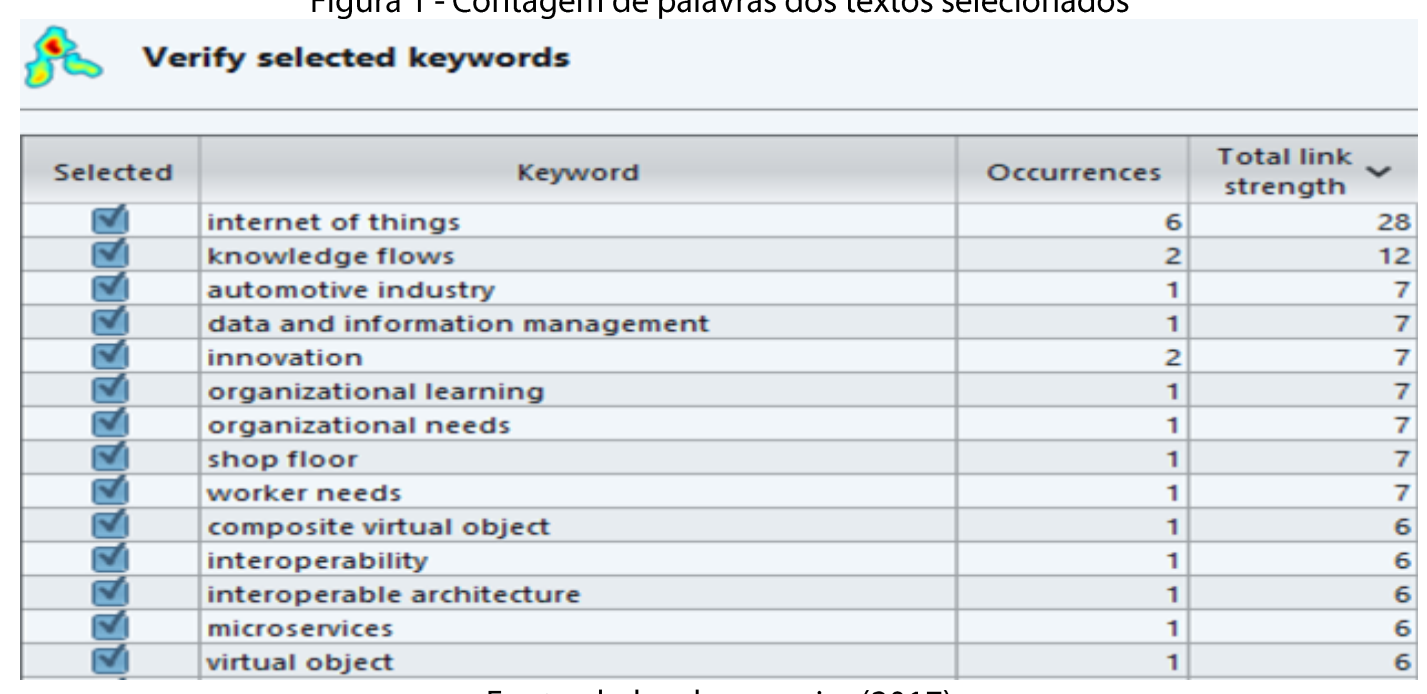

Fonte: dados da pesquisa (2017)

A palavra internet of things (internet das coisas) possui maior ocorrência, afirmando a lei de Zipf. Isso pode ocorrer também devido a elevada publicação encontrada nos resultados individuais e relacionais por pares quando o tema é internet das coisas. Também foi possível desenvolver um mapa empírico da densidade dos termos com ocorrência. A escala de ocorrência foi colocada como 15 vezes (FIGURA 2). 
Figura 2- Mapa empírico de densidade de termos com ocorrência superior a quinze vezes

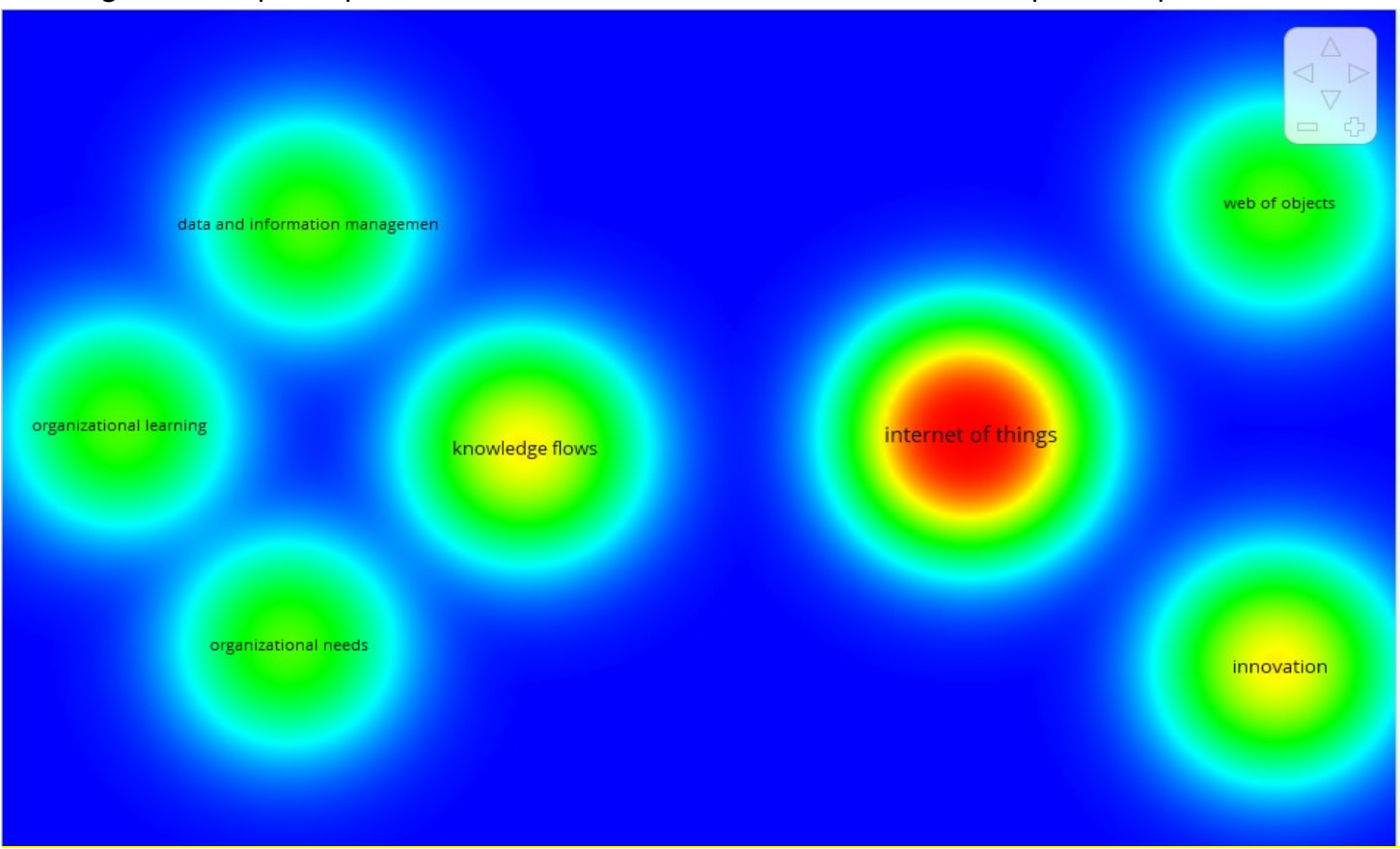

Fonte: dados da pesquisa (2017)

Mais uma vez, a maior ocorrência está na palavra internet of things. Isso vem afirmar a ideia de que a Internet das Coisas tem evoluído e, com isso, despertado a atenção de pesquisadores. Esse tema é crescente e promissor, tanto para a academia quanto para o mercado.

\subsection{A busca de autores, periódicos e áreas em ênfase}

Para situar as referências sobre "Gestão do Conhecimento", "Inovação" e "Internet das Coisas" na comunidade científica, esse trabalho utilizou indicadores bibliográficos estatísticos de dados quantitativos encontrados na produção técnica e científica para identificar os autores mais relevantes, baseados na evolução histórica do número de publicações (FIGURA 3).

Figura 3 - Autores mais relevantes citados nos trabalhos obtidos

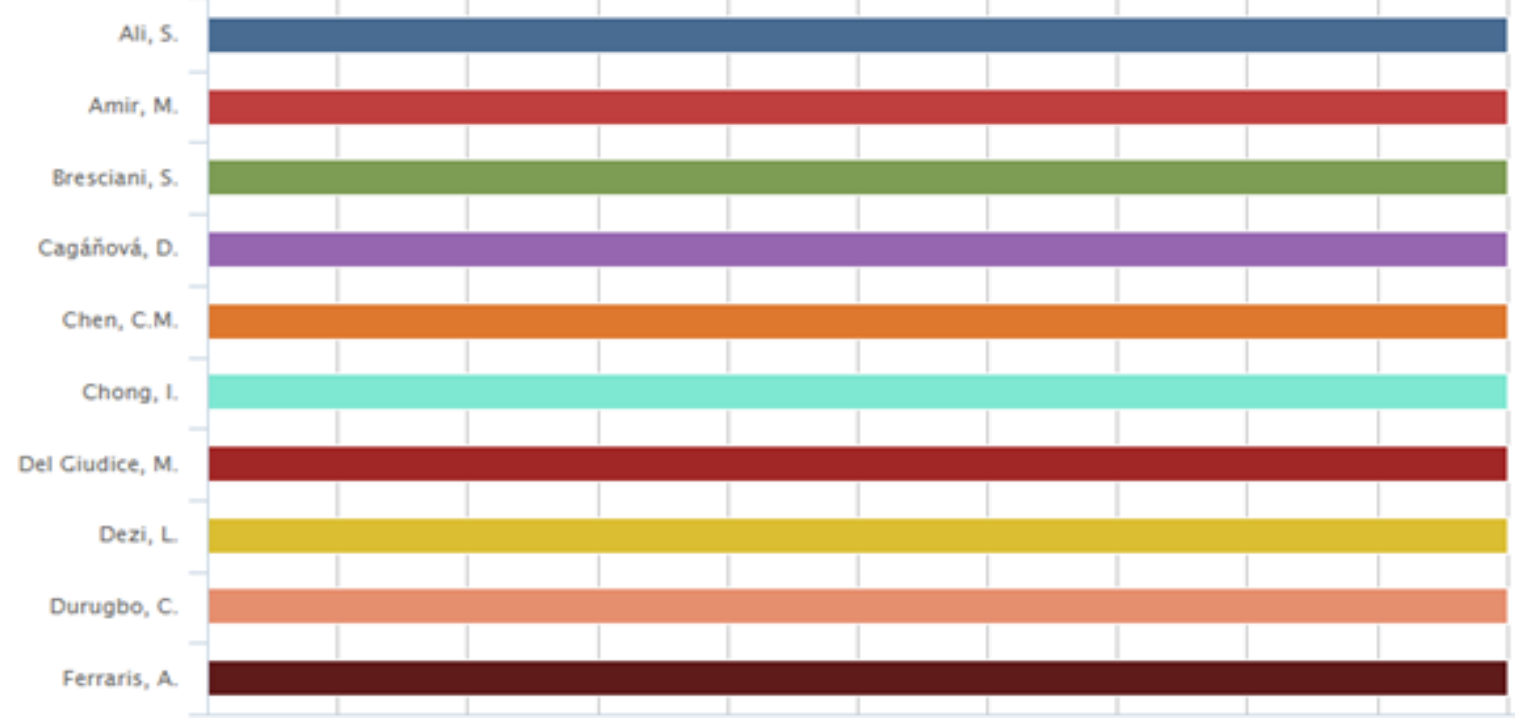

Fonte: dados da pesquisa (2017) 
Identificou-se que os autores Ali, s. Amir, M., Bresciani. S, Coganová, D., Chen, C.M. Chong, I. Del Giudice, M., Dezi, L. Durugbo, c. e Ferraris, A. são os mais citados. Em continuidade, buscou-se identificar o período com o maior número de publicações e o índice de impacto. O índice de impacto das revistas científicas tem sido usado para avaliá-las, bem como a qualidade da pesquisa científica. Esse parâmetro está institucionalizado pela CAPES como um critério importante para avaliação de periódicos e, por isso, foi utilizado para esse estudo.

Nesse artigo, utiliza-se as métricas quantitativas CiteScore da entidade Scopus Elsevier, por serem abrangentes, transparentes, atuais e gratuitas. Encontrou-se o Lecture Notes in Computer Science como resultado do periódico com maior número de publicações e que possui um índice de impacto satisfatório. $O$ resultado remete a outros pensamentos que podem aprimorar e valorizar as pesquisas que relacionem os três temas propostos.

Nas pesquisas brasileiras, os conhecimentos são organizados em áreas de concentração e linhas de pesquisa. Área de concentração é onde se limita certos ramos de conhecimentos, atividades ou competências. Segundo Erdmann e Lanzoni (2008) refere-se ao universo de disciplinas e interdisciplinar, à organização das mesmas em especialidades ou processo de viver, processo saúde e doença, dentre outras, e a possibilidade de classificação para visibilidade mediante nomenclatura internacional.

A Figura 4 apresenta as principais áreas de concentrações das publicações analisadas.

Figura 4 - Principais áreas de concentração das publicações

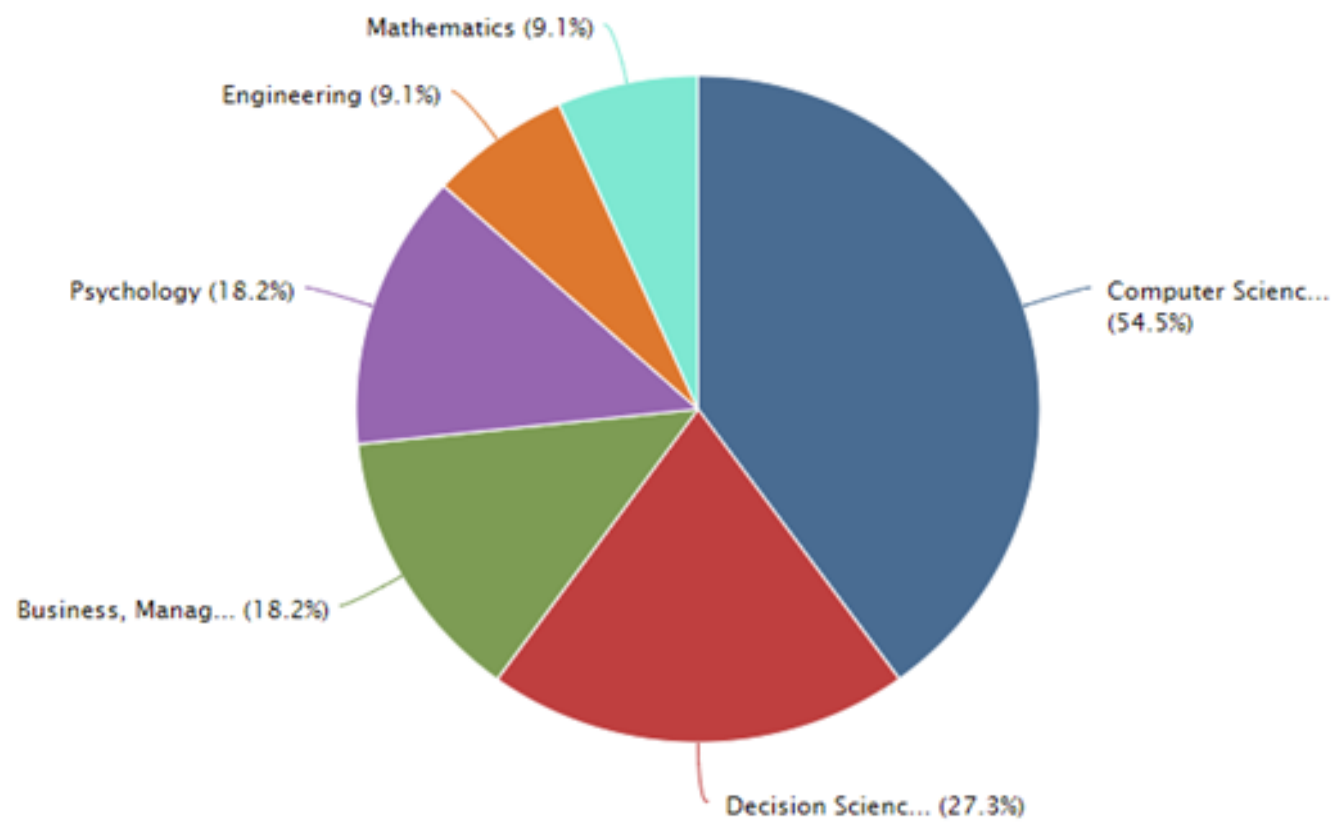

Fonte: dados da pesquisa (2017)

As áreas de maior concentração das publicações dos termos gestão do conhecimento, internet das coisas e inovação está na ciência da computação e ciência de decisão. O resultado sobre as áreas de concentração das pesquisas pode ser reflexo da própria origem da "internet das coisas", haja vista que o conceito de "Coisa" tem evoluído junto à tecnologia, visando a colheita de informações e a interação com o mundo físico, conforme proposto por Singer (2012) e Asthon (2009).

Um resultado interessante está na Psicologia como área principal no desenvolvimento de pesquisas desses constructos. Apesar de serem temas geralmente relacionados à tecnologia ou administração, observase a extensão das áreas e da interdisciplinaridade da gestão do conhecimento. 


\section{CONSIDERAÇõES FINAIS}

Esse estudo teve como objetivo identificar a intensidade das possíveis relações entre os constructos Gestão do Conhecimento, Inovação, Internet das coisas. Essa intensidade foi analisada por meio de uma revisão sistemática da literatura na Base Capes. Em complementariedade, buscou-se identificar as principais palavras citadas nos textos, os principais autores, periódico e áreas de concentração que se empenham nos estudos da relação dos constructos propostos.

A escolha por pesquisar a relação entre as temáticas de Gestão do Conhecimento, Internet das Coisas e Inovação, vem da importância que cada temática possui de maneira isolada no atual cenário e do carácter integrador que a Gestão do Conhecimento demostra sobre as duas temáticas. A pesquisa caracterizou-se como descritiva, de abordagem quantitativa e foi realizada uma análise dos trabalhos obtidos na revisão sistemática.

Foi possível identificar que, ao se pesquisar os constructos separadamente, um número considerável de publicações científicas é retornado. De maneira isolada, o constructo Internet das Coisas, apresenta um maior número de publicações. Esse fato não ocorre quando os constructos são relacionados por pares e trios. Ao se analisar as pesquisas relacionando os temas, o número de artigos declina consideravelmente, totalizando apenas 20 artigos capazes de relacionar a gestão do conhecimento, internet das coisas e inovação.

Pode-se ressaltar que o objetivo geral da pesquisa foi alcançado, bem como os objetivos específicos. As principais palavras citadas, os principais autores que relacionam os temas, o principal período de publicação e as áreas de concentração, foram identificadas. A pesquisa contribuiu para semear os conceitos de Gestão do Conhecimento, Internet das Coisas e Inovação, principalmente enfatizando a existência de uma lacuna na integração dos temas. Ao mesmo tempo, comprovou-se a importância desse artigo e sua interdisciplinaridade.

O estudo se limitou a realizar uma análise quantitativa das publicações que falam das três temáticas em conjunto e se concentrou na base CAPES. Assim uma sugestão de pesquisa futura seria analisar qualitativamente os trabalhos obtidos em uma revisão sistemática sobre as três temáticas e incluir a Tecnologia de Informação como constructo relacional.

\section{REFERÊNCIAS}

ASHTON, K. That 'Internet of Things' thing. RFID Journal, June, 2009. Disponível em: <http://www.rfidjournal.com/articles/view?4986>. Acesso em: 10. jun. 2017.

BAGLIERI, E. Dall'idea al valore [From idea to value]. Milano: Etas Libri, 2003.

BARBOSA, Ricardo Rodrigues. Gestão da informação e do conhecimento: origens, polêmicas e perspectivas. Informação \& Informação, Londrina, v. 13, n. 1 esp, p. 1-25, 2008.

BAREGHEH, A.; ROWLEY, J.; SAMBROOK, S. Towards a multidisciplinary definition of innovation. Management Decision, v. 47, n. 8, p. 1323-1339, 2009. http://dx.doi. Org/10.1108/00251740910984578

BERNARDES, R.; ANDREASSI, T. Inovação em serviços intensivos em conhecimento. São Paulo: Saraiva, 2007.

GUEDES, Vânia L.S.; BORSCHIVER, Suzana. Bibliometria: uma ferramenta estatística para a gestão da informação e do conhecimento, em sistemas de informação, de comunicação e de avaliação científica e tecnológica. [2005]. Disponível em: <www.cinform-anteriores.ufba.br/vi_anais/docs/VaniaLSGuedes.pdf>. Acesso em: 19 jun. 2018.

CASTRO, A. A. et al. Curso de revisão sistemática e metanálise. São Paulo: LED-DIS/UNIFESP: 2006. 
CHOO, C. W. A Organização do Conhecimento. São Paulo: SENAC, 2003.

DAVENPORT, T. H.; PRUSAK, L. Conhecimento empresarial: como as organizações gerenciam o seu capital intelectual. 6. ed. Rio de Janeiro: Campus, 1998.

ERDMANN, A. L.; LANZONI, G. M. M. Características dos grupos de pesquisa de enfermagem brasileira certificados pelo CNPQ de 2005 a 2007. Esc Anna Nery Rev Enferm, v. 12, n. 2, p. 316-322, 2008.

FERRAZ, E. Internet das Coisas. In: MOSTRA DE INICIAÇÃO CIENTIFICA, 11., 2016, Bagé, RS. Anais do II CONGREGA DE ÉTICA E INOVAÇÃO SOCIAL. Bagé: UFRGS, 2016. p. 42-49.

FREEMAN, C; SOLET, L. The economics of industrial innovation. Londres: Pinter, 1982.

GALVÃO, C. M.; SAWADA, N. O.; TREVIZAN, M. A. Revisão sistemática: recurso que proporciona a incorporação das evidências na prática da enfermagem. Revista Latino-Americana de Enfermagem, Ribeirão Preto, v. 12, n. 3, p. 549-556, maio/jun. 2004.

GUBBI, J.; BUYYA, R.; MARUSIC, S.; PALANISWAMI, M. Internet of Things: A vision, architectural elements and future directions. Future Generation Computer Systems, Vol. 29, Issue 7, Sept. 2013. Disponível em: <http://www.sciencedirect.com/science/article/pii/S0167739X13000241>. Acesso em: 05 set. 2017.

LOPES, Daniel Paulino Teixeira et al. Inovação: conceitos, metodologias e aplicabilidade. Articulando um construto à formulação de políticas públicas. Uma reflexão sobre a lei de inovação de Minas Gerais. In. SEMINAR ON THE ECONOMY OF MINAS, 13., 2008, Belo Horizonte. Anais dos 13th Seminar on the Economy. Belo Horizonte: UFMG, 2008. p. 234-250.

YAMAKAWA, E. K. et al. Comparativo dos softwares de gerenciamento de referências bibliográficas: Mendeley, EndNote e Zotero. Transinformação, v. 26, n. 2, 2014.

MUYLDER, C. F. et al. International Jornal Knowledge. Engineering and Management, Florianópolis, v. 3, n.7, p. 153-170, nov. 2014/fev. 2015.

NONAKA, l.; TAKEUCHI, H. Criação de conhecimento na empresa: como as empresas japonesas geram a dinâmica da inovação. 19. ed. Rio de Janeiro: Elsevier, 1997.

NORD, W.; TUCKER, S. Implementing Routine and Radical Innovations. Lexington: Lexington Books, 1987.

PAIVA, Simone Bastos. Modelo conceitual para o capital intelectual integrado a Gestão do Conhecimento. Informação \& Sociedade, v. 22, 2012.

PERISSÉ, A.R.S.; GOMES M.M.; NOGUEIRA S.A. Revisões sistemáticas (inclusive metanálises) e diretrizes clínicas. In: GOMES, M.M. (Org.). Medicina baseada em evidências: princípios e práticas. Rio de Janeiro, RJ: Reichmann \& Affonso; 2001. p.131-148.

PERROTTI, E.; VASCONCELLOS, E. P. G. Estrutura Organizacional e Gestão do Conhecimento. Revista Eletrônica de Ciência Administrativa (RECADM), v. 4, n. 2, 2005. p. 1-18.

PINHEIRO, A. O. M. Tecnologia de Informação e Comunicação (TIC), Inovação e serviços intensivos em conhecimento: o que os indicadores retratam e o que poderiam revelar. Tese (Doutorado) - Universidade Federal do Rio de Janeiro, 2011.

SCHUMPETER, J. A Teoria do desenvolvimento econômico. São Paulo: Abril Cultural 1981. [Coleção Os Economistas].

SINGER, Talita. Tudo conectado: conceitos e representações da internet das coisas. Simpósio em tecnologias digitais e sociabilidade, v. 2, p. 1-15, 2012. 
SILVA, L. N. A quarta onda. 4. ed. Rio de Janeiro: Record, 1995.

SOUZA, T. L. M. et al. Desenvolvimento de competências estratégicas para inovação: evidências a partir do caso do setor automotivo brasileiro. In: ENCONTRO NACIONAL DE ENGENHARIA DE PRODUÇãO- Inovação Tecnologia e Propriedade Intelectual: Desafios da Engenharia de Produção na Consolidação do Brasil no Cenário Econômico Mundial. 31, 2011, Belo Horizonte. Anais do XXXI Encontro Nacional de Engenharia de Produção. Belo Horizonte: UFMG, 2011. p. 1-13.

TERRA, J. C. 10 Dimensões da gestão da inovação. Rio de Janeiro: Elsevier, 2012.

TIDD, J.; BESSANT, J.; PAVITT, K. Gestão da inovação. 3. ed. Porto Alegre: Bookman, 2008.

TIGRE, P. B. Gestão da inovação: a economia da tecnologia no Brasil. Rio de Janeiro: Campus, 2006.

TOFFLER, Alvin. The third wave. New York: Bantam books, 1980.

TSOUKAS, H.; VLADIMIROU, E. What is organizational knowledge? Journal Management Studies, v. 38, n. 7, p. 973-993, 2001. 